\title{
基于小波特征参数的平面四杆机构轨迹综合方法
}

\author{
刘文瑞 ${ }^{1}$ 孙建伟 ${ }^{1,2}$ 褚金奎 ${ }^{3}$ \\ (1. 长春工业大学机电工程学院 长春 130012; \\ 2. 吉林大学工程仿生教育部重点实验室 长春 130022; \\ 3. 大连理工大学机械工程学院 大连 116024)
}

\begin{abstract}
摘要: 小波分解可以在不同尺度下对函数曲线的特征进行提取, 基于该理论, 首先, 对平面四杆机构连杆轨迹曲线进行小波 分解, 利用归一化处理方法提取轨迹曲线的小波特征参数, 给出了平面四杆机构连杆轨迹曲线的小波特征参数近似描述方法。 进而结合数值图谱法, 将 11 维非整周期轨迹综合问题转换为 6 维机构尺寸型检索问题, 同时建立包含 3004281900 组平面 四杆机构的机构尺寸型数据库。根据轨迹曲线小波特征参数的特点, 利用多维搜索树, 将各相对转动区间内的机构尺寸型进 行分区, 建立索引关键字数据库。从而通过比较给定轨迹曲线小波特征参数与索引关键字, 查找并提取目标机构所在叶子结 点中机构尺寸型的小波特征参数, 建立自适应图谱库。在此基础上, 根据给定设计要求的小波特征参数与自适应图谱库中的 小波特征参数的相似程度, 检索出满足设计要求的机构尺寸型。再根据理论公式计算目标机构的实际尺寸及安装位置, 实现 平面四杆机构非预定相对转动区间轨迹综合问题的求解。最后, 利用小波特征参数法对滚压包边设备的滚轮进给机构进行设 计, 验证本方法实用性和有效性的同时, 为传统滚压包边提供新的思路。
\end{abstract}

关键词: 小波特征参数; 平面四杆机构; 多维搜索树; 自适应图谱库; 非整周期轨迹综合

中图分类号: TH122

\section{Synthesis Method for Path Generation of a Planar Four-bar Mechanism Based on the Wavelet Feature Parameters}

\author{
LIU Wenrui $^{1}$ SUN Jianwei ${ }^{1,2}$ CHU Jinkui ${ }^{3}$
}

(1. School of Mechatronic Engineering, Changchun University of Technology, Changchun 130012;

2. Key Laboratory for Terrain-machine Bionics Engineering, Jilin University, Changchun 130022;

3. School of Mechanical Engineering, Dalian University of Technology, Dalian 116024)

\begin{abstract}
Wavelet function can extract the feature information of function curve in different scales. Based on the wavelet transform theory, a coupler curve generated by planar four-bar mechanism is decomposed to wavelet coefficients. A normalised process is proposed for extracting wavelet feature parameters. Then a description method for coupler curve of planar four-bar mechanism is established using wavelet feature parameters. The eleven dimensional open path synthesis problems are transformed to six basic dimensional types searching problems. A database is established, which contains 3004281900 groups of dimensional types. The dimensional types are decomposed by using multidimensional search tree and an index keyword database is established. The leaf node which contains the desired mechanism can be obtained. A self-adaptive atlas database is established. Based on the design requirements, the desired mechanism can be obtained by comparing the similarity of wavelet feature parameters between the prescribed curve and the coupler curve in the self-adaptive atlas database. According to the internal relationship of wavelet coefficients, the actual sizes and installation positions of the desired linkage can be calculated. The arbitrary relative rotation interval open path synthesis is realized. Two examples are presented to demonstrate the effectiveness of the method proposed. Particularly, a roller feed mechanism for roller hemming is designed and manufactured to verify the validity and practicality of this method. The design results provide new ideas for the roller hemming.
\end{abstract}

Key words: wavelet feature parameters; planar four-bar mechanism; multidimensional search tree; self-adaptive atlas database; open path synthesis

* 国家自然科学基金资助项目(51775054)。20180822 收到初稿, 20181208 收到修改稿 


\section{0 前言}

连杆机构尺度综合作为一个经典问题, 一直是 机构学领域的研究热点。国内外众多学者提出了多 种能够实现预期轨迹的连杆机构尺寸设计方法 ${ }^{[1]}$ 。 数值图谱法作为传统图谱法的分支, 其基本思路是： 首先, 将大量轨迹曲线的特征进行参数化处理, 如 利用 $\mathrm{B}$ 样条曲线 ${ }^{[2]}$ 、傅里叶级数 ${ }^{[3-4]}$ 、小波系数 ${ }^{[5]}$ 、 轨迹曲线曲率半径及相对转角 ${ }^{[6-7]}$ 等方法提取连杆 机构轨迹曲线的特征参数。再将特征参数储存在图 谱库中。最后, 根据给定的设计要求, 对目标机构 进行匹配识别, 检索出满足设计要求的连杆机构尺 寸型。

UNRUH 等 ${ }^{[8]}$ 利用周期 $\mathrm{B}$ 样条曲线对平面四杆 机构输出轨迹曲线进行描述。结合数值图谱法, 实 现了平面四杆机构整周期多位置点的轨迹综合问题 的求解。黄灿明等 ${ }^{[9]}$ 将优化算法与数值图谱法相结 合, 基于 $\mathrm{B}$ 样条插值曲线理论, 提出了一种先近似 检索, 再精确匹配的四杆机构轨迹综合方法。于红 英等 ${ }^{[10]}$ 利用三次非均匀 $\mathrm{B}$ 样条曲线对连杆轨迹曲 线进行拟合并提取特征参数。将特征参数与基本尺 寸型(四杆杆长)及连杆上任意一点的位置参数储存 在一起, 建立数值图谱库。再通过人工神经网络在 数值图谱库中匹配目标机构尺寸型。由于上述方法 屏蔽了机架位置、机架偏转角度及机构整体缩放对 特征参数的影响, 从而有效降低了机构尺寸型数据 库的维度, 减少了图谱库中的数据冗余。但是, 由 于连杆上任意一点位置参数的改变对特征参数存在 影响, 利用上述方法进行轨迹综合时, 需要将位置 参数作为独立变量存储在数据库中, 从而导致所建 立的数值图谱库中涵盖的机构基本尺寸型较少, 占 用空间较大, 综合结果精度不高。

MCGARVA 等 ${ }^{[11-12]}$ 利用傅里叶级数对四杆机构 轨迹曲线进行描述, 并给出了各项谐波特征参数的 几何意义。褚金奎等 ${ }^{[13-14]}$ 对连杆机构轨迹综合问题 进行了深入研究, 通过分析发现, 对于整周期轨迹 曲线, 利用五项谐波特征参数可以近似描述机构轨 迹曲线特点, 进而建立了连杆机构输出轨迹曲线的 谐波特征参数数据库。基于平面四杆机构轨迹综合 的研究成果, MULLINEUX ${ }^{[15]}$ 将傅里叶级数法推广 到球面四杆机构尺度综合中。上述基于傅里叶级数 的连杆机构尺度综合方法中, 所提取的谐波特征参 数不仅与机架位置、机架偏转角度及机构整体缩放 比例无关, 还与连杆上任意一点的位置参数无关。 与基于 B 样条曲线的连杆机构轨迹综合方法相比,
傅里叶级数法所建立的特征参数数据库中只存储基 本尺寸型和五项谐波特征参数。相同条件下，傅里 叶级数法所建立的数值图谱库中涵盖的机构尺寸型 更多, 综合结果的精度更高。傅里叶级数法可以很 好地实现整周期给定设计要求的连杆机构轨迹综 合, 但是利用傅里叶级数法求解非整周期尺度综合 问题时, 需要对给定的非整周期设计要求补充若干 采样点, 再根据拓展得到的整周期设计条件, 利用 建立的数值图谱库进行尺度综合。该方法只能使设 计结果对拓展后的整周期设计要求进行整体逼近, 无法保证对特定相对转动区间进行逼近。针对这一 问题, $\mathrm{WU}$ 等 ${ }^{[16]}$ 利用傅里叶级数理论对非整周期设 计要求的平面四杆机构轨迹综合问题进行了深入的 研究, 给出了同时适用于整周期和非整周期设计要 求的轨迹综合方法。在此基础上, YUE 等 ${ }^{[17]}$ 将基于 傅里叶级数的非整周期设计要求尺度综合方法进一 步完善, 利用 $P$ 型傅里叶级数描述四杆机构输出轨 迹曲线, 并开发了一套能够实现非整周期设计要求 的计算机辅助设计软件。然而, 由于傅里叶级数的 自身局限性, 基于傅里叶级数的非整周期尺度综合 方法所得到的设计结果往往精度不高, 并且综合过 程耗时较长。

随着小波理论的日益完善, 其可在不同尺度下 对曲线的近似分量和细节分量进行特征提取的特点 受到广泛关注，基于小波特征参数的尺度综合方法 也逐渐发展起来。吴金等 ${ }^{[18]}$ 将小波特征参数法引入 到平面四杆机构函数综合中, 利用 Daubechies 小波 描述了平面四杆输出函数曲线的特征。王成志等 ${ }^{[19]}$ 利用 Haar 小波对平面四杆机构连杆轨迹曲线进行 特征提取, 将 64 种典型四杆机构杆长组合与 180 个连杆上任意一点位置参数相结合, 建立了包括 11520 组机构尺寸参数的数值图谱库。GALÁNMARÍN 等 ${ }^{[20]}$ 将连杆机构轨迹曲线的 Daubechies 小 波描述方法与神经网络识别方法相结合, 提出了一 种适用于非均匀时标设计条件的整周期轨迹综合方 法。SUN 等 ${ }^{[21]}$ 将小波特征参数法推广到空间连杆机 构非整周期尺度综合问题中。上述方法可以很好地 解决给定相对转动区间的尺度综合问题, 但是在实 际问题中，相对转动区间往往是未知的。由于相对 转动区间是独立变量, 采用上述设计方法对未给定 相对转动区间的尺度综合问题进行求解的过程中, 需要人为预定相对转动区间, 无法保证预定的相 对转动区间内存在近似最优解 ${ }^{[22]}$ 。针对这一问题, 本文提出了基于小波特征参数的非预定相对转动区 间轨迹综合方法。利用预处理、小波分解、归一化 处理方法对四杆机构轨迹参数进行降维, 消除了机 
架位置、机架偏转角度、机构整体缩放以及连杆上 任意一点位置变化对小波特征参数的影响。利用多 维搜索树建立了平面四杆机构自适应图谱库。根据 给定设计条件的小波特征参数与自适应图谱库中所 存储的小波特征参数的相似程度, 输出多组机构尺 寸型。利用遗传算法对所得综合结果进行优化。再 根据理论公式计算目标机构的实际尺寸及安装位置 参数。实现非预定相对转动区间轨迹综合问题的求 解。与基于 $\mathrm{B}$ 样条曲线的轨迹综合方法相比 ${ }^{[2,8-10]}$, 本文所提出的小波特征参数法屏蔽了连杆上任意一 点位置参数对特征参数的影响, 从而减少了图谱库 中的数据冗余, 增加了图谱库中涵盖的机构尺寸型 数量, 提高了综合精度; 与基于傅里叶级数的轨迹 综合方法相比 ${ }^{[11-17]}$, 小波特征参数法对非整周期轨 迹曲线的描述更加准确, 弥补了傅里叶级数法无法 对特定相对转动区间进行尺度综合的不足。

\section{1 平面四杆机构连杆轨迹曲线的小波 特征参数}

\section{1 平面四杆机构连杆轨迹曲线的数学模型}

一般安装位置的平面四杆机构如图 1 所示, 其 中: $L_{1}$ 为输入构件 $A B$ 的长度, $L_{2}$ 为连杆 $B C$ 的长 度, $L_{3}$ 为连架杆 $C D$ 的长度, $L_{4}$ 为机架 $A D$ 的长度; $P$ 为连杆上任意一点, $L_{P}$ 为 $B P$ 的长度, $\theta_{P}$ 为 $B P$ 与连杆 $B C$ 之间的夹角; $L_{A}$ 为坐标原点 $O$ 与点 $A$ 之 间的距离, $\theta_{A}$ 为 $O A$ 与 $x$ 轴的夹角; $\theta_{0}$ 为机架 $A D$ 与 $x$ 轴的夹角; $\theta_{t}$ 为机架 $A D$ 与输入构件 $A B$ 的夹角; $\theta_{2}$ 为机架 $A D$ 与连杆 $B C$ 的夹角; $\theta^{\prime}{ }_{1}$ 为机构起始角 度; $\theta_{1}$ 为输入转角。

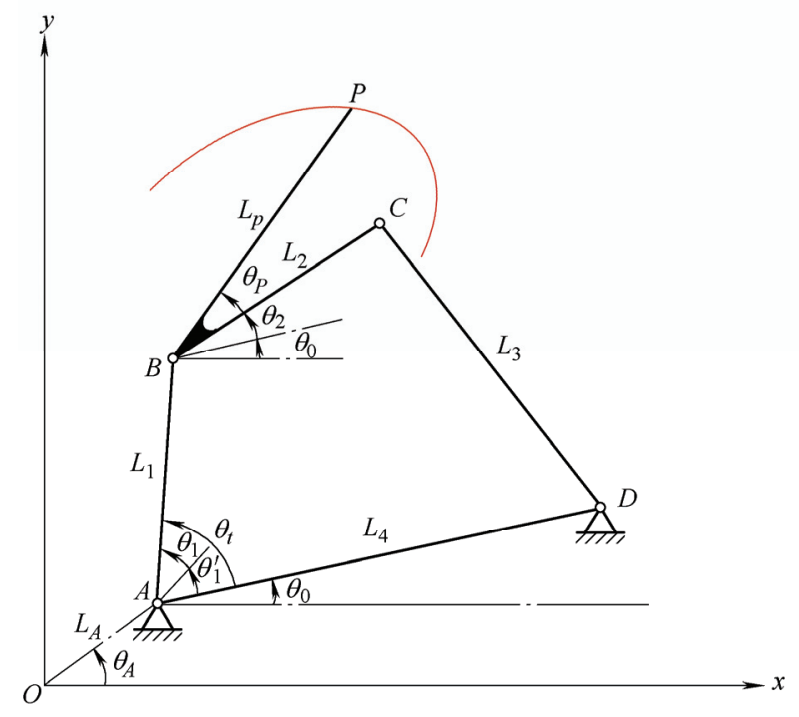

图 1 一般位置平面四杆机构
在复平面上, $P$ 点的轨迹曲线可以表示为

$$
\begin{gathered}
\boldsymbol{P}=L_{A} \exp \left(\mathrm{i} \theta_{A}\right)+L_{1} \exp \left[\mathrm{i}\left(\theta_{0}+\theta_{t}\right)\right]+ \\
L_{p} \exp \left[\mathrm{i}\left(\theta_{0}+\theta_{2}+\theta_{P}\right)\right]
\end{gathered}
$$

式中, $\theta_{t}=\theta_{1}{ }^{\prime}+\theta_{1}, 0<\theta_{1} \leqslant \theta_{s}\left(\theta_{s}\right.$ 为输入构件相对起 始角的最大转动角度)。本文定义输入构件相对起始 角的转动范围 $\left(\theta_{1}\right.$ 的取值范围)为相对转动区间。根 据几何关系可知,连杆 $B C$ 与机架 $A D$ 之间的夹角 $\theta_{2}$ 不受机架安装位置及安装角度的影响, 从而, 可以 根据标准安装位置四杆机构模型计算 $\theta_{2}$ 角 ${ }^{[23]}$ 。图 2 为标准安装位置的平面四杆机构, 其中: 点 $A$ 与坐 标原点 $O$ 重合; 杆 $A D$ 与 $x$ 轴重合; $B G$ 与 $A D$ 平行; 点 $E$ 为 $B E$ 与 $A D$ 的垂足; 点 $F$ 为 $C F$ 与 $B D$ 的垂足; 点 $G$ 为 $C G$ 与 $B G$ 的垂足; $L_{A E}$ 为 $A E$ 长度; $L_{B E}$ 为 $B E$ 长度; $L_{B F}$ 为 $B F$ 长度; $L_{C F}$ 为 $C F$ 长度; $L_{B G}$ 为 $B G$ 长度; $L_{C G}$ 为 $C G$ 长度; $L_{B D}$ 为 $B D$ 长度; $\theta_{D B C}$ 为 $B D$ 与 $B C$ 的夹角; $\theta_{B D A}$ 为 $B D$ 与 $D A$ 的夹角。根 据三角关系, $\theta_{2}$ 可由 $L_{A E} 、 L_{B E} 、 L_{B D} 、 \theta_{B D A} 、 \theta_{D B C}$ 、 $L_{B G} 、 L_{C G}$ 表示, 具体理论公式如下 ${ }^{[24]}$ 。

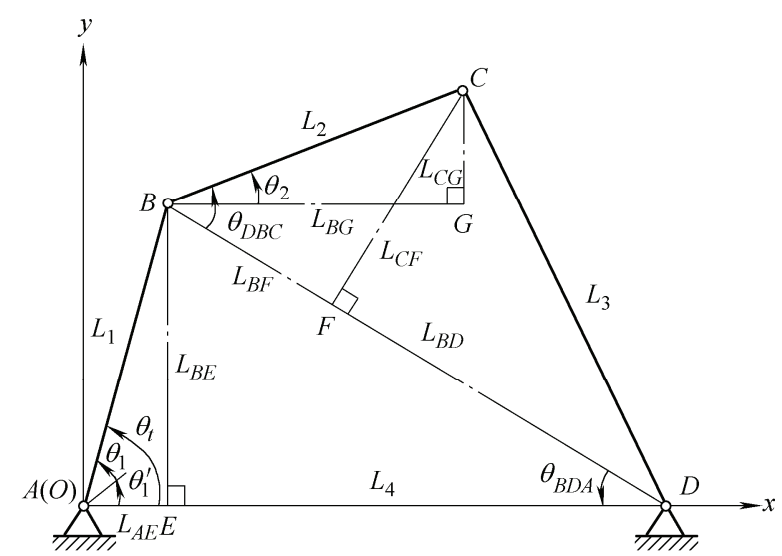

图 2 标准安装位置四杆机构

(1) $A E$ 长度 $L_{A E}$ 和 $B E$ 长度 $L_{B E}$

$$
\begin{aligned}
& L_{A E}=L_{1} \cos \theta_{t} \\
& L_{B E}=L_{1} \sin \theta_{t}
\end{aligned}
$$

(2) $B D$ 长度 $L_{B D}$

$$
L_{B D}=\sqrt{\left(L_{4}-L_{A E}\right)^{2}+L_{B E}^{2}}
$$

(3) $B D$ 与 $D A$ 的夹角 $\theta_{B D A}$

$$
\theta_{B D A}=\arctan \left[L_{B E} /\left(L_{4}-L_{A E}\right)\right]
$$

(4) $B D$ 与 $B C$ 的夹角 $\theta_{D B C}$

$$
\theta_{D B C}=\arccos \left[\left(L_{B D}{ }^{2}+L_{2}{ }^{2}-L_{3}^{2}\right) / 2 L_{B D} L_{2}\right]
$$

(5) $B G$ 长度 $L_{B G}$ 和 $C G$ 长度 $L_{C G}$

$$
L_{B G}=L_{B F} \cos \theta_{B D A}+L_{C F} \sin \theta_{B D A}=
$$




$$
\begin{gathered}
L_{2} \cos \theta_{D B C} \cos \theta_{B D A}+L_{2} \sin \theta_{D B C} \sin \theta_{B D A} \\
L_{C G}=L_{C F} \cos \theta_{B D A}-L_{B F} \sin \theta_{B D A}= \\
L_{2} \sin \theta_{D B C} \cos \theta_{B D A}-L_{2} \cos \theta_{D B C} \sin \theta_{B D A}
\end{gathered}
$$

根据几何关系， $\theta_{2}$ 可以表示为

$$
\theta_{2}=\arctan \left(L_{C G} / L_{B G}\right)
$$

将式(2) (8) 代入式(9)得

$$
\begin{aligned}
& \theta_{2}=\arctan \left[\frac{\left(a+b \cos \theta_{t}\right) \sin \theta_{t}+c\left(L_{4}-L_{1} \cos \theta_{t}\right)}{d+e \cos \theta_{t}+b \cos ^{2} \theta_{t}+c L_{1} \sin \theta_{t}}\right] \\
& a=-L_{1}\left(L_{1}{ }^{2}+L_{2}^{2}-L_{3}^{2}+L_{4}^{2}\right) \\
& b=2 L_{1}^{2} L_{4} \\
& c=\left\{4 L_{2}^{2}\left(L_{1}^{2}+L_{4}^{2}\right)-\left(L_{1}^{2}+L_{2}^{2}-L_{3}^{2}+L_{4}^{2}\right)^{2}-\right. \\
& {\left[8 L_{1} L_{2}^{2} L_{4}-4 L_{1} L_{4}\left(L_{1}{ }^{2}+L_{2}{ }^{2}-L_{3}{ }^{2}+L_{4}{ }^{2}\right)\right] \times} \\
& \left.\cos \theta_{t}-4 L_{1}^{2} L_{4}^{2} \cos ^{2} \theta_{t}\right\}^{1 / 2} \\
& d=L_{4}\left(L_{1}^{2}+L_{2}^{2}-L_{3}^{2}+L_{4}^{2}\right) \\
& e=-L_{1}\left(L_{1}{ }^{2}+L_{2}{ }^{2}-L_{3}{ }^{2}+L_{4}{ }^{2}\right)-2 L_{1} L_{4}{ }^{2}
\end{aligned}
$$

根据式(1)和式(10)可知, 连杆上任意一点 $P$ 的 轨迹曲线可由 11 个参数确定, 即 $L_{1}, L_{2}, L_{3}, L_{4}$, $L_{P}, L_{A}, \theta_{P}, \theta_{A}, \theta_{0}, \theta_{1}^{\prime}, \theta_{s}$ 。本文定义这些参数为 四杆机构轨迹参数。

在利用数值图谱法求解四杆机构轨迹综合的问 题中, 所建立的数据库中尺寸型维度越多, 数据的 存储量及匹配识别的计算量越大。若将四杆机构轨 迹参数全部作为机构尺寸型与其对应的小波系数直 接存储在一起建立输出特征数据库, 会造成建立的 数据库所包含的数据量过于庞大, 机构尺寸型圥余, 匹配识别时间长, 综合效率低等问题。本文利用预 处理和小波变换的方法, 在不减少机构输出种类的 前提下, 达到剔除数据库中重复尺寸型的目的。

\section{2 平面四杆机构非整周期轨迹曲线的预处理}

根据数值图谱法求解连杆机构尺度综合问题的 一般思路, 首先, 对连杆轨迹曲线进行离散化采样。 令输入构件以 $\theta^{\prime}{ }_{1}$ 为起始角, $\omega$ 为角速度匀速转动。 根据式(1), 四杆机构连杆轨迹曲线的第 $m$ 个采样点 可以表示为 $\left(m \in \mathbf{N}^{*}\right)$

$$
\begin{gathered}
\boldsymbol{P}\left(t_{m}\right)=L_{A} \exp \left(i \theta_{A}\right)+L_{1} \exp \left\{i\left[\theta_{0}+\theta_{1}^{\prime}+\theta_{1}\left(t_{m}\right)\right]\right\}+ \\
L_{p} \exp \left\{i\left[\theta_{0}+\theta_{2}\left(t_{m}\right)+\theta_{P}\right]\right\}
\end{gathered}
$$

式中, $t_{m}$ 为连杆上任意一点 $P$ 转动到第 $m$ 个采样点 的时刻; $\theta_{1}\left(t_{m}\right)$ 为第 $m$ 个时刻的输入角 $\left(\theta_{1}\left(t_{m}\right)=\omega t_{m}\right.$, $\left.m=0,1, \cdots, 2^{j}, j \in \mathbf{N}^{*}\right), \theta_{2}\left(t_{m}\right)$ 为第 $m$ 时刻连杆与机 架的夹角。

对轨迹曲线进行预处理, 即, 将所有轨迹点逆 时针旋转 $\Delta \theta\left(\Delta \theta=\theta_{s} / 2^{j}\right)$, 再用原曲线上第 $n(n=1$, $\left.2, \cdots, 2^{j}\right)$ 个采样点 $\boldsymbol{P}\left(t_{n}\right)$ 减去旋转变换后的第 $n-1$ 个 采样点 $\exp (\mathrm{i} \Delta \theta) \boldsymbol{P}\left(t_{n-1}\right)$, 所得矢量可以表示为

$$
\boldsymbol{E}\left(t_{n}\right)=\boldsymbol{P}\left(t_{n}\right)-\exp (\mathrm{i} \Delta \theta) \boldsymbol{P}\left(t_{n-1}\right)
$$

将式(11)代入式(12)中，得

$$
\begin{gathered}
\boldsymbol{E}\left(t_{n}\right)=L_{A} \exp \left(\mathrm{i} \theta_{A}\right)[1-\exp (\mathrm{i} \Delta \theta)]+ \\
L_{p} \exp \left[\mathrm{i}\left(\theta_{0}+\theta_{P}\right)\right] \boldsymbol{\theta}_{2}^{n} \\
\boldsymbol{\theta}_{2}^{n}=\exp \left[\mathrm{i} \theta_{2}\left(t_{n}\right)\right]-\exp \left\{\mathrm{i}\left[\theta_{2}\left(t_{n-1}\right)+\Delta \theta\right]\right\}
\end{gathered}
$$

根据式(13)可知, 预处理后得到的矢量包含了 所有四杆机构轨迹参数。在保证四杆机构轨迹参数 信息完整的前提下, 预处理可以为利用小波分解理 论提取轨迹曲线的小波特征参数提供基础。本文定 义预处理后得到的矢量为曲线特征矢量。

\section{3 平面四杆机构连杆轨迹曲线的小波特征参数}

根据式(13)，任意四杆机构轨迹曲线可以用一 组曲线特征矢量 $\boldsymbol{E}\left(t_{1}\right), \boldsymbol{E}\left(t_{2}\right), \cdots, \boldsymbol{E}\left(t_{2^{j}}\right)$ 表示。根据小 波分解理论, 利用 Haar 小波对曲线特征矢量进行小 波分解, 曲线特征矢量的一级小波分解可表示为

$$
\boldsymbol{E}=\boldsymbol{E}_{1}+\boldsymbol{W}_{1}
$$

$\boldsymbol{E}_{1}=\sum_{l=1}^{2^{j-1}}\left[\frac{\boldsymbol{E}\left(t_{2 l-1}\right)+\boldsymbol{E}\left(t_{2 l}\right)}{2} h_{\varphi}\left(2^{j-1} \frac{t-t_{1}}{t_{s}}-l+1\right)\right]$

$\boldsymbol{W}_{1}=\sum_{l=1}^{2^{j-1}}\left[\frac{\boldsymbol{E}\left(t_{2 l-1}\right)-\boldsymbol{E}\left(t_{2 l}\right)}{2} h_{\psi}\left(2^{j-1} \frac{t-t_{1}}{t_{s}}-l+1\right)\right]$

式中, $l=1,2, \cdots, 2^{j-1}, t_{s}=\theta_{s} / \omega, h_{\varphi}$ 为尺度函数, $h_{\psi}$ 为小波函数, 它们可以表示为

$$
\begin{gathered}
h_{\varphi}\left(2^{j-1} \frac{t-t_{1}}{t_{s}}-l+1\right)= \\
\begin{cases}1 & 0 \leqslant 2^{j-1} \frac{t-t_{1}}{t_{s}}-l+1<1 \\
0 & \text { 其他 }\end{cases} \\
h_{\psi}\left(2^{j-1} \frac{t-t_{1}}{t_{s}}-l+1\right)= \\
\left\{\begin{array}{cc}
1 & 0 \leqslant 2^{j-1} \frac{t-t_{1}}{t_{s}}-l+1<\frac{1}{2} \\
-1 & \frac{1}{2} \leqslant 2^{j-1} \frac{t-t_{1}}{t_{s}}-l+1<1 \\
0 & \frac{\text { 其他 }}{0}
\end{array}\right.
\end{gathered}
$$

根据小波多分辨率分析理论及递推关系

$$
\begin{gathered}
\boldsymbol{E}_{J-1}=\boldsymbol{E}_{J}+\boldsymbol{W}_{J} \\
\boldsymbol{E}_{J}=\sum_{l=1}^{2^{j-J}}\left[\frac{\boldsymbol{E}\left(t_{2^{J} l-2^{J}+1}\right)+\cdots+\boldsymbol{E}\left(t_{2^{J} l}\right)}{2^{J}} \times\right.
\end{gathered}
$$




$$
\begin{gathered}
\left.h_{\varphi}\left(2^{j-J} \frac{t-t_{1}}{t_{s}}-l+1\right)\right] \\
\boldsymbol{W}_{J}=\sum_{l=1}^{2^{j-J}}\left\{\left[\boldsymbol{E}\left(t_{2^{J} l-2^{J}+1}\right)+\cdots+\boldsymbol{E}\left(t_{2^{J} l-2^{J-1}}\right)-\right.\right. \\
\left.\boldsymbol{E}\left(t_{2^{J} l-2^{J-1}+1}\right)-\cdots-\boldsymbol{E}\left(t_{2^{J} l}\right)\right] / 2^{J} \times \\
\left.h_{\psi}\left(2^{j-J} \frac{t-t_{1}}{t_{s}}-l+1\right)\right\}
\end{gathered}
$$

式中 $J=2,3, \cdots, j, l=1,2, \cdots, 2^{j-J}$ 。对式(15)继续 分解得曲线特征矢量的二级小波分解

$$
\boldsymbol{E}=\boldsymbol{E}_{2}+\boldsymbol{W}_{2}+\boldsymbol{W}_{1}
$$

曲线特征矢量的三级小波分解

$$
\boldsymbol{E}=\boldsymbol{E}_{3}+\boldsymbol{W}_{3}+\boldsymbol{W}_{2}+\boldsymbol{W}_{1}
$$

曲线特征矢量的 $j$ 级小波分解

$$
\boldsymbol{E}=\boldsymbol{E}_{j}+\sum_{J=1}^{j} \boldsymbol{W}_{J}
$$

根据式(21) (23), 曲线特征矢量的 $j$ 级小波分 解可以表示为

$$
\boldsymbol{E}=\boldsymbol{a}_{(j, 1)} h_{\varphi(j, 1)}+\sum_{J=1}^{j} \sum_{l=1}^{2^{j-J}}\left[\boldsymbol{d}_{(J, l)} h_{\psi(J, l)}\right]
$$

式中, $\boldsymbol{a}_{(j, 1)}$ 为 $j$ 级小波平均数 $\left(j \in \mathbf{N}^{*}\right), \boldsymbol{d}_{(J, l)}$ 为第 $J$ 级 第 $l$ 个小波细节数 $\left(J=1,2, \cdots, j, l=1,2, \cdots, 2^{j-J}\right)$, 它们可以表示为

$$
\begin{aligned}
& \boldsymbol{a}_{(j, 1)}=\frac{\boldsymbol{E}\left(t_{1}\right)+\cdots+\boldsymbol{E}\left(t_{2^{j}}\right)}{2^{j}} \\
& \boldsymbol{d}_{(J, l)}=\frac{\left[\boldsymbol{E}\left(t_{2^{J} l-2^{J}+1}\right)+\cdots+\boldsymbol{E}\left(t_{2^{J} l-2^{J-1}}\right)\right]}{2^{J}}- \\
& \frac{\left[\boldsymbol{E}\left(t_{2^{J} l-2^{J-1}+1}\right)+\cdots+\boldsymbol{E}\left(t_{2^{J} l}\right)\right]}{2^{J}} \\
& h_{\varphi(j, 1)}=h_{\varphi}\left(\frac{t-t_{1}}{t_{s}}\right)=\left\{\begin{array}{cc}
1 & 0 \leqslant \frac{t-t_{1}}{t_{s}}<1 \\
0 & \text { 其他 }
\end{array}\right. \\
& h_{\psi(J, l)}=h_{\psi}\left(2^{j-J} \frac{t-t_{1}}{t_{s}}-l+1\right)= \\
& \left\{\begin{array}{cc}
1 & 0 \leqslant 2^{j-J} \frac{t-t_{1}}{t_{s}}-l+1<\frac{1}{2} \\
-1 & \frac{1}{2} \leqslant 2^{j-J} \frac{t-t_{1}}{t_{s}}-l+1<1 \\
0 & \text { 其他 }
\end{array}\right.
\end{aligned}
$$

对小波细节数进行归一化处理, 即, 所有小波细节 数除以第 $j$ 级小波细节数, 得

$$
\begin{gathered}
\boldsymbol{b}_{(J, l)}=\frac{\boldsymbol{d}_{(J, l)}}{\boldsymbol{d}_{(j, 1)}}=2^{j-J}\left\{\left[\boldsymbol{E}\left(t_{2^{J} l-2^{J}+1}\right)+\cdots+\right.\right. \\
\left.\left.\boldsymbol{E}\left(t_{2^{J} l-2^{J-1}}\right)\right]-\left[\boldsymbol{E}\left(t_{2^{J} l-2^{J-1}+1}\right)+\cdots+\boldsymbol{E}\left(t_{2^{J} l}\right)\right]\right\} / \\
\left\{\left[\boldsymbol{E}\left(t_{1}\right)+\cdots+\boldsymbol{E}\left(t_{2^{j-1}}\right)\right]-\left[\boldsymbol{E}\left(t_{2^{j-1}+1}\right)+\cdots+\boldsymbol{E}\left(t_{2^{j}}\right)\right]\right\}
\end{gathered}
$$

将式(13)代入式(29)中得

$$
\begin{gathered}
\boldsymbol{b}_{(J, l)}=2^{j-J}\left\{\left[\boldsymbol{\theta}_{2}^{2^{J} l-2^{J}+1}+\cdots+\boldsymbol{\theta}_{2}^{2^{J} l-2^{J-1}}\right]-\right. \\
\left.\left[\boldsymbol{\theta}_{2}^{2^{J} l-2^{J-1}+1}+\cdots+\boldsymbol{\theta}_{2}^{2^{J} l}\right]\right\} /\left\{\left[\boldsymbol{\theta}_{2}^{1}+\cdots+\boldsymbol{\theta}_{2}^{2^{j-1}}\right]-\right. \\
\left.\left[\boldsymbol{\theta}_{2}^{2^{j-1}+1}+\cdots+\boldsymbol{\theta}_{2}^{2^{j}}\right]\right\}
\end{gathered}
$$

本文定义归一化处理后的小波细节数为小波特 征参数。根据式(10)、式(14)、式(30)可知, 连杆轨 迹曲线的小波特征参数只与 $\theta^{\prime}{ }_{1}, \theta_{s}$ 及基本尺寸型 $\left(L_{1}, L_{2}, L_{3}, L_{4}\right)$ 有关, 本文定义这些参数为机构尺 寸型。除机构尺寸型外, 其他四杆机构轨迹参数的 改变对小波特征参数没有影响。根据这一发现, 可 以将四杆机构轨迹综合过程分为两步: 首先, 建立 机构尺寸型数据库，根据给定设计要求的小波特征 参数与机构尺寸型的小波特征参数的相似度, 在数 据库中匹配识别出目标机构的机构尺寸型; 然后, 根据给定设计要求的小波系数与匹配识别出的目标 机构尺寸型的小波系数之间的内在联系, 利用理论 公式计算目标机构实际尺寸及安装位置。上述方法 可以将机构尺寸型相同的四杆机构输出轨迹用同一 组小波特征参数表示。从而实现在有限的存储空间 中, 将机构尺寸型相同的四杆机构聚类储存, 消除 机构尺寸型数据冗余的目的。

\section{2 平面四杆自适应图谱库的建立及 轨迹综合方法}

根据小波多分辨率分析理论，小波变换通过伸 缩和平移小波函数对目标函数曲线进行逐步多尺度 细化, 利用小波系数描述函数曲线与尺度函数和小 波函数的相似程度, 实现特征信息提取 ${ }^{[25]}$ 。根据复 数运算关系可知, 对曲线特征矢量进行小波变换相 当于对曲线特征矢量实部和虚部分别进行小波变 换。图 3 为四杆机构曲线特征矢量实部的一维小波 分解示意图, 其中, $\operatorname{Re}(\boldsymbol{E})$ 表示轨迹曲线特征矢量实 部; $\operatorname{Re}(\boldsymbol{a})$ 表示轨迹曲线特征矢量实部的小波平均 数; $\operatorname{Re}(\boldsymbol{d})$ 表示轨迹曲线特征矢量实部的小波细节 数。根据小波分解理论可知, 小波平均数可以描述 曲线在当前尺度下的大体趋势，小波细节数可以描 
述曲线在当前尺度下的变化情况。由于四杆机构输 出轨迹曲线连续且平滑 (不考虑尖点问题), $j-1$ 级小 波特征参数 $\left(\boldsymbol{b}_{(j-1,1)}\right.$ 和 $\left.\boldsymbol{b}_{(j-1,2)}\right)$ 可以表示曲线在低分辨 率下的走势和特征。根据这一特点, 本文建立了包 含 3004281900 组机构尺寸型的数据库, 具体步骤 如下。

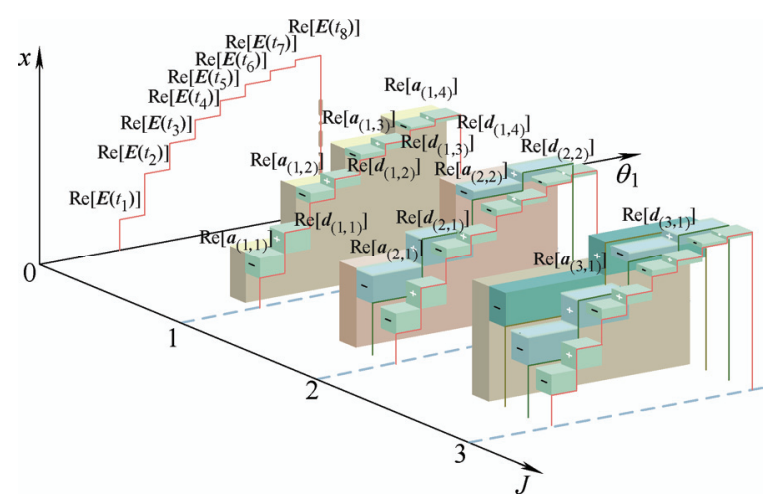

图 3 一维小波分解示意图

（1）令四杆机构各杆长的初始值为 1 , 步长为 3 , 四杆杆长总和为 1000 , 机构起始角度为 $1^{\circ}$, 步长为 $2^{\circ}$, 相对转动区间为 $31^{\circ} \sim 299^{\circ}$, 变化步长为 $2^{\circ}$, 建立机构尺寸型数据库。根据格拉斯霍夫准则, 满 足要求的基本尺寸型有 123633 组, 所建立的机构 尺寸型数据库中共包含 3004281900 组机构尺寸 型, 其中, 每个相对转动区间包含 22253940 组机 构尺寸型。

（2）根据第 1.3 节的分析可知, 除机构尺寸型外 的其他四杆机构轨迹参数的变化对小波特征参数没 有影响, 为了方便计算实际尺寸和安装位置, 本文 令: $L_{P}=1, L_{A}=0, \theta_{P}=0, \theta_{A}=0, \theta_{0}=0$ 。将这些 参数与机构尺寸型结合, 得到平面四杆机构轨迹参 数。对所有机构尺寸型生成的连杆机构的轨迹曲线 进行预处理、小波变换及归一化处理, 将得到的 $j-1$ 级小波特征参数 $\left(\boldsymbol{b}_{(j-1,1)}\right.$ 和 $\left.\boldsymbol{b}_{(j-1,2)}\right)$ 与相应的机构尺寸 型存储于小波特征参数数据库中。

(3) 利用多维搜索树, 对相对转动区间内的机 构尺寸型进行分区, 建立索引关键字数据库 ${ }^{[26-27]}$ 。 首先, 根据小波特征参数中第一项的实部, 将机构 尺寸型排序, 建立深度为 2 的 4 阶搜索树, 其中, 子树节点的关键字为小波特征参数中第一项实部所 构成序列的四等分点数值(如图 4 所示, 其中, $k w$ 为关键字; $\operatorname{Re}\left[\boldsymbol{b}_{(j-1,1)}\right]$ 表示第 $j-1$ 级第 1 个小波特征 参数的实部; $\operatorname{Im}\left[\boldsymbol{b}_{(j-1,1)}\right]$ 表示第 $j-1$ 级第 1 个小波特 征参数的虚部; $\operatorname{Re}\left[\boldsymbol{b}_{(j-1,2)}\right]$ 表示第 $j-1$ 级第 2 个小波 特征参数的实部; $\operatorname{Im}\left[\boldsymbol{b}_{(j-1,2)}\right]$ 表示第 $j-1$ 级第 2 个小 波特征参数的虚部)。再根据第一项小波特征参数的 虚部、第二项小波特征参数的实部和虚部的数值,
对子树进行划分。最终, 得到深度为 5 的 4 阶搜索 树, 其中, 叶子结点数为 256 个, 每个节点存储约 87000 个机构尺寸型编号。索引关键字数据库中包 含 135 组相对转动区间的索引关键字序列, 每个序 列包括 255 个关键字。

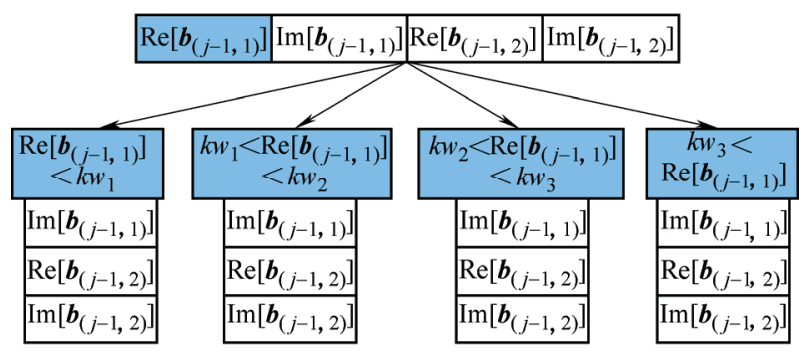

图 4 深度为 2 的多维搜索树构建

(4) 根据给定设计要求, 利用小波特征参数法 提取设计要求的 $j-1$ 级小波特征参数。通过与索引 关键字数据库中的关键字进行比对, 查询得到目标 机构尺寸型所在叶子结点。根据多尺度分析理论, 结合机构尺寸型数据库, 提取叶子结点中全部机构 尺寸型生成四杆机构的小波特征参数, 建立自适应 图谱库。自适应图谱库中每组数据包括机构尺寸型 编号和相应的小波特征参数。上述方法实现了以设 计要求为条件的动态自适应图谱库的建立。

在利用自适应图谱库进行尺度综合的过程中， 只需要机构尺寸型数据库、索引关键字数据库和叶 子结点, 就可以实现平面四杆机构非预定相对转动 区间轨迹综合问题的求解。由于每个叶子结点只存 储约 87000 个机构尺寸型编号, 每个尺寸型编号占 用 4 个字符, 经过压缩后, 每个叶子节点仅占用约 $200 \mathrm{kB}$ 的磁盘空间。与传统数值图谱法相比, 自适 应图谱库的建立时间、占用磁盘空间及目标机构的 匹配识别时间远远少于传统数值图谱法。根据多分 辨率分析理论及四杆机构输出轨迹的特点可知, 利 用 $j-1$ 级和 $j-2$ 级小波特征参数可以近似描述曲线的 特点。本文所建立的自适应图谱库中只保存六项小 波特征参数, 这样在不影响综合精度的同时, 可以 进一步提高匹配效率。

根据自适应图谱库中的小波特征参数与给定设 计要求的小波特征参数的相似程度, 对目标机构的 机构尺寸型进行匹配识别, 在自适应图谱库中检索 出满足设计要求的机构尺寸型。相似度函数为

$$
\delta=\sum_{J=j-2}^{j-1} \sum_{l=1}^{2^{j-J}}\left[\left(\boldsymbol{b}_{(J, l)}-\boldsymbol{b}_{(J, l)}^{\prime}\right)^{2}\right]
$$

式中, $\boldsymbol{b}_{(J, l)}$ 为给定设计要求的 $J$ 级小波特征参数, $\boldsymbol{b}_{(J, l)}^{\prime}$ 为机构尺寸型生成机构的 $J$ 级小波特征参数。根据 给定设计要求的小波系数与所得目标机构的小波系 
数之间的内在联系, 计算目标机构的实际尺寸及安 装位置, 具体的理论公式如下。

(1) $B P$ 长度 $L_{P}$

$$
L_{p}=\sqrt{\left\{\operatorname{Re}\left[\boldsymbol{d}_{(j, 1)} / \boldsymbol{d}_{(j, 1)}^{\prime}\right]\right\}^{2}+\left\{\operatorname{Im}\left[\boldsymbol{d}_{(j, 1)} / \boldsymbol{d}_{(j, 1)}^{\prime}\right]\right\}^{2}}
$$

式中, $\boldsymbol{d}_{(j, 1)}$ 为给定设计要求的 $j$ 级小波细节数, $\boldsymbol{d}_{(j, 1)}^{\prime}$ 为机构尺寸型生成机构的 $j$ 级小波细节数。

(2) 机架位置 $L_{A}$ 和 $\theta_{A}$

$$
\begin{gathered}
L_{A}=\sqrt{[\operatorname{Re}(\boldsymbol{c})]^{2}+[\operatorname{Im}(\boldsymbol{c})]^{2}} \\
\theta_{A}=\arctan [\operatorname{Im}(\boldsymbol{c}) / \operatorname{Re}(\boldsymbol{c})] \\
\boldsymbol{c}=\frac{\boldsymbol{a}_{(j, 1)}-\boldsymbol{a}_{(j, 1)}^{\prime} L_{P} \exp \left[\mathrm{i}\left(\theta_{P}+\theta_{0}\right)\right]}{1-\exp (\mathrm{i} \Delta \theta)}
\end{gathered}
$$

式中, $\boldsymbol{a}_{(j, 1)}$ 为给定设计要求的 $j$ 级小波平均数, $\boldsymbol{a}_{(j, 1)}^{\prime}$ 为机构尺寸型生成机构的 $j$ 级小波平均数, $\theta_{P}+\theta_{0}$ 可由下式表示

$$
\begin{gathered}
\theta_{P}+\theta_{0}=\arctan [\operatorname{Im}(\boldsymbol{e}) / \operatorname{Re}(\boldsymbol{e})] \\
\boldsymbol{e}=\boldsymbol{d}_{(j, 1)} / \boldsymbol{d}_{(j, 1)}^{\prime}
\end{gathered}
$$

（3）机构实际杆长 $L_{1}, L_{2}, L_{3}, L_{4}$

$$
L_{M}=k L_{M}^{\prime}
$$

式中, $M=1,2,3,4, L^{\prime}{ }_{M}$ 为机构尺寸型的杆长, 比 例系数 $k$ 可由下式给出

$$
\begin{gathered}
k=\left\{\left[\operatorname { R e } \left(\boldsymbol{P}\left(t_{1}\right)-L_{p} \exp \left(\mathrm{i}\left(\theta_{0}+\theta_{2}\left(t_{1}\right)+\theta_{P}\right)\right)-\right.\right.\right. \\
\left.\left.L_{A} \exp \left(\mathrm{i} \theta_{A}\right)\right)\right]^{2}+\left[\operatorname { I m } \left(\boldsymbol{P}\left(t_{1}\right)-L_{p} \exp \left(\mathrm { i } \left(\theta_{0}+\right.\right.\right.\right. \\
\left.\left.\left.\left.\left.\theta_{2}\left(t_{1}\right)+\theta_{P}\right)\right)-L_{A} \exp \left(\mathrm{i} \theta_{A}\right)\right)\right]^{2}\right\}^{1 / 2} / L_{1}^{\prime}
\end{gathered}
$$

(4) 机架偏转角度 $\theta_{0}$ 及 $B P$ 与连杆夹角 $\theta_{P}$

$$
\begin{gathered}
\theta_{0}=\arctan \left\{\operatorname { I m } \left[\boldsymbol{P}\left(t_{1}\right)-L_{p} \exp \left(\mathrm { i } \left(\theta_{0}+\theta_{2}\left(t_{1}\right)+\right.\right.\right.\right. \\
\left.\left.\left.\theta_{P}\right)\right)-L_{A} \exp \left(\mathrm{i} \theta_{A}\right)\right] / \operatorname{Re}\left[\boldsymbol{P}\left(t_{1}\right)-L_{p} \exp \left(\mathrm { i } \left(\theta_{0}+\right.\right.\right. \\
\left.\left.\left.\left.\theta_{2}\left(t_{1}\right)+\theta_{P}\right)\right)-L_{A} \exp \left(\mathrm{i} \theta_{A}\right)\right]\right\}-\theta_{1}^{\prime}
\end{gathered}
$$

式中, $\boldsymbol{P}\left(t_{1}\right)$ 为给定设计要求的第一个采样点, $\theta_{2}\left(t_{1}\right)$ 为第一个采样点对应的连杆与机架夹角。将所得 $\theta_{0}$ 代入式(34), 可以得到 $B P$ 与连杆夹角 $\theta_{P}$ 。

根据式(31) (37), 可以得到目标机构的所有轨 迹参数。由于数据库中的机构尺寸型是离散的; 同 时, 在利用多维搜索树检索目标机构尺寸型的过程 中, 没有进行回溯, 针对这一问题, 本文利用遗传 算法对所得机构尺寸型进行优化, 在所得机构尺寸 型附近搜索更优解。将优化后的轨迹参数作为设计 结果输出, 最终实现平面四杆机构非预定相对转动 区间轨迹综合问题求解。

\section{3 算例}

\section{1 对比算例}

为验证上述理论方法的正确性，本文以文献[22] 中例 2 所给出的直线轨迹作为设计条件，对平面四 杆机构进行轨迹综合。目标轨迹为: 从坐标点 $(0.5,0)$ 到坐标点 $(0.93,0.25)$ 的直线。由于文献[22]中所提出 的方法只能在特定区间进行轨迹综合，所以文献[22] 中人为预定了相对转动区间: $\theta_{1} \in\left[0,135^{\circ}\right]$ 。所得最 优综合结果的平均欧氏距离误差为 $1.8093 \times 10^{-3} \mathrm{~m}$ 。 利用本文所提出的方法, 对相同目标轨迹进行综合, 所得结果列于表 1 , 图 5 为第一组综合结果与目标 轨迹曲线的对比图及误差图。在与文献[22]近似相 同的计算环境下，自适应图谱库的建立及匹配识别 共用时 $335.472046 \mathrm{~s}$, 比文献[22]的匹配识别时间少 $259.427301 \mathrm{~s}$ 。由于本文所提出的方法是在非预定 相对转动区间的条件下进行特征提取及匹配识别, 所得综合结果的近似最优相对转动区间约为 $30^{\circ}$, 平均欧式距离误差为 $1.5734 \times 10^{-6} \mathrm{~m}$, 综合结果精度 比文献[22]提高了 1000 倍。

表 1 对比算例综合结果

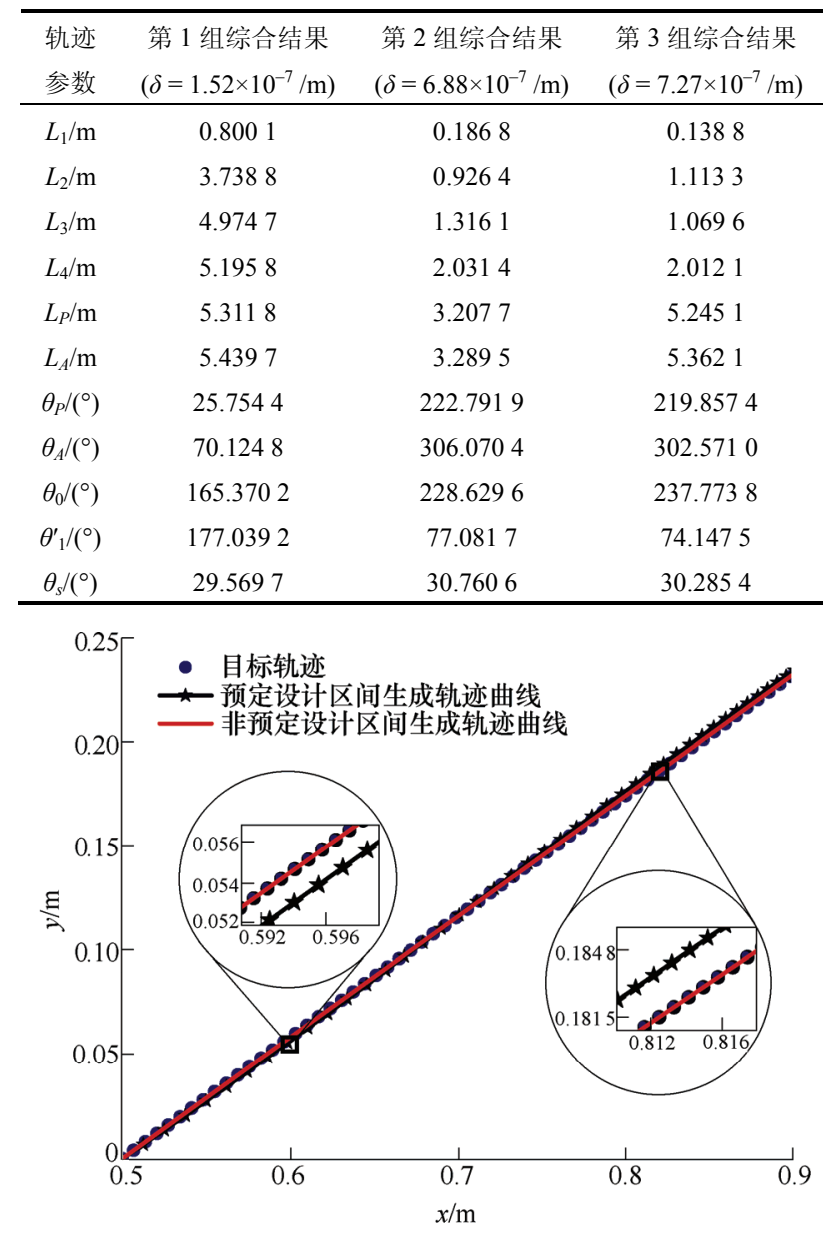

(a) 综合结果对比图 


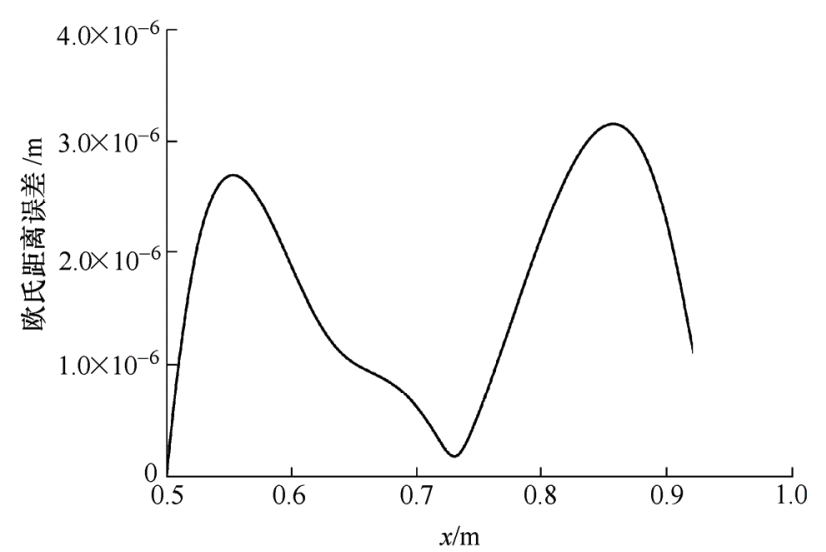

(b) 综合结果误差图

图 5 对比算例第一组综合结果对比图及误差图

通过与文献[22]的综合结果比较可以发现, 相 对转动区间对综合结果的影响较大, 在轨迹综合问 题中, 相对转动区间往往是未知的, 虽然设计人员 可以通过曲线形状及相关经验对目标机构的相对转 动区间进行预估，但无法保证预定的相对转动区间 内存在近似最优解。如利用文献[22]提出的方法建 立与本文相同大小(包含 135 个相对转动区间)的数 值图谱库, 需要约 $134 \mathrm{~GB}$ 的磁盘空间, 建库及匹 配时间超过 $120000 \mathrm{~s}$ 。而本文所提出的轨迹综合方 法可以根据设计条件快速建立自适应图谱库, 进而, 可以在 135 个相对转动区间中对目标机构进行匹配 识别。相比于文献[22]中只能在单一相对转动区间 内进行匹配识别检索, 本文所提的方法在轨迹综合 问题的求解上具有独特的优势。

\section{2 应用算例}

基于四杆机构对滚压包边设备的滚轮进给机构 进行设计。如图 6 所示, 滚轮进给机构的一个铰点 与十字滑台连接, 另一铰点与伺服电动机连接, 通 过何服电动机带动输入构件在特定区间内转动, 从 而实现滚轮在滚压包边路径上的滚压。所用伺服电 动机为台达 ECMA-C10807RS, 额定功率为 $750 \mathrm{~W}$, 额定扭矩为 $2.39 \mathrm{~N} \cdot \mathrm{m}$, 额定输出转速为 $3000 \mathrm{r} / \mathrm{min}$ 。 为增加通用性, 将杆件制作成长度可调部件, 通过 调整十字滑台及杆件上滑块的位置实现对安装位置 及杆件长度的调整(十字滑台的有效行程为 350 $\mathrm{mm})$ 。

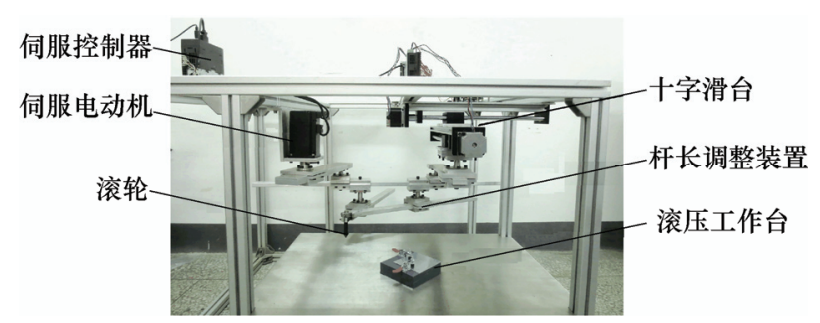

图 6 滚轮进给机构
以图 7 所示曲线为滚压包边路径，利用小波特 征参数法对滚轮进给机构的杆件尺寸及安装位置进 行设计, 所得结果列于表 2 , 其中, 第一组综合结 果的欧式距离误差最小(图 8)。为验证方法的有效性 和模型的正确性, 利用 CATIA V5R20 软件对综合结 果进行模拟仿真。首先, 根据第一组综合结果对机 构模型进行设计和装配。机构模型中各杆件尺寸 及机构位置参数为: $L_{1}=276.1527, L_{2}=380.4440$, $L_{3}=382.1508, L_{4}=452.0838, L_{P}=139.0157, L_{A}=$ $417.7083, \theta_{P}=280.2402^{\circ}, \theta_{A}=78.5461^{\circ}, \theta_{0}=$ $259.3438^{\circ}$ 。然后, 利用 CATIA 软件中的 DMU 模块 对装配模型进行仿真。根据综合结果, 设置主动件

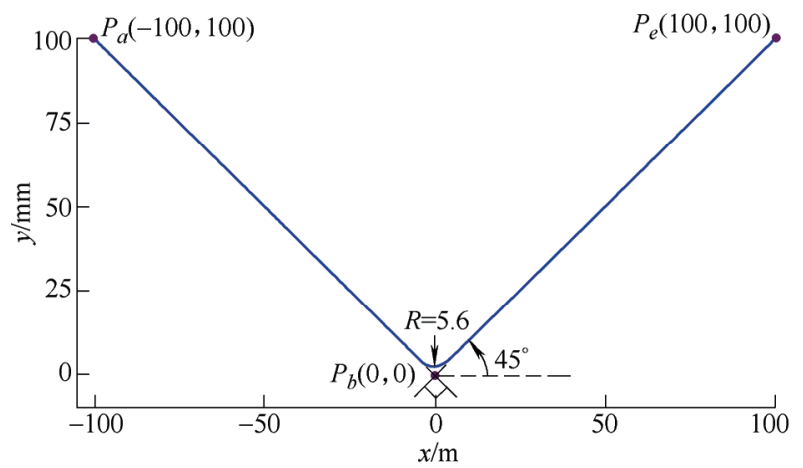

图 7 滚压包边路径

表 2 应用算例综合结果

\begin{tabular}{cccc}
\hline 轨迹 & 第 1 组综合结果 & $\begin{array}{c}\text { 第 } 2 \text { 组综合结果 } \\
\left(\delta=6.52 \times 10^{-3} / \mathrm{mm}\right)\end{array}$ & $\begin{array}{c}\text { 第3 组综合结果 } \\
\left(\delta=6.51 \times 10^{-3} / \mathrm{mm}\right)\end{array}$ \\
\hline$L_{1} / \mathrm{mm}$ & 276.1527 & 278.8354 & 272.8513 \\
$L_{2} / \mathrm{mm}$ & 380.4440 & 413.4807 & 365.0213 \\
$L_{3} / \mathrm{mm}$ & 382.1508 & 414.8182 & 387.1568 \\
$L_{4} / \mathrm{mm}$ & 452.0838 & 456.8578 & 447.7096 \\
$L_{P} / \mathrm{mm}$ & 139.0157 & 140.7919 & 135.0560 \\
$L_{A} / \mathrm{mm}$ & 417.7083 & 421.9513 & 410.1939 \\
$\theta_{P} /\left(^{\circ}\right)$ & 280.2402 & 279.5672 & 277.3818 \\
$\theta_{A} /\left(^{\circ}\right)$ & 78.5461 & 79.3383 & 77.8272 \\
$\theta_{0} /\left(^{\circ}\right)$ & 259.3438 & 260.1016 & 257.1745 \\
$\theta_{1}^{\prime} /\left(^{\circ}\right)$ & -40.3353 & -40.2077 & -38.8822 \\
$\theta_{S} /\left(^{\circ}\right)$ & 80.9109 & 80.5995 & 80.9113 \\
\hline
\end{tabular}

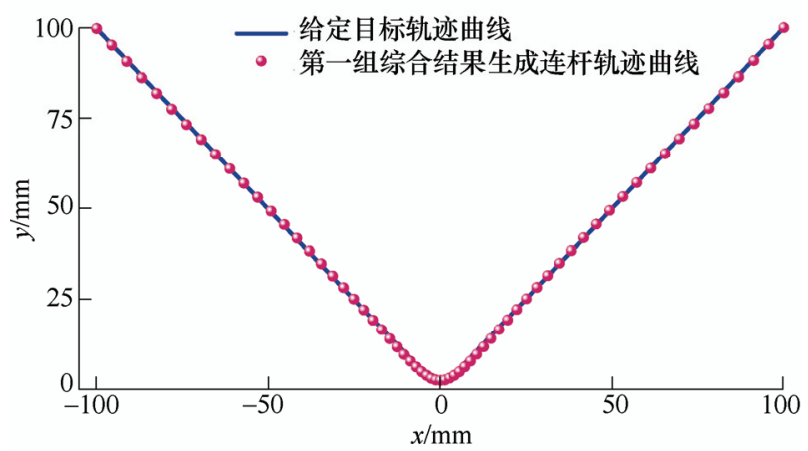

(a) 目标轨迹曲线与第一组综合结果生成连杆轨迹曲线 


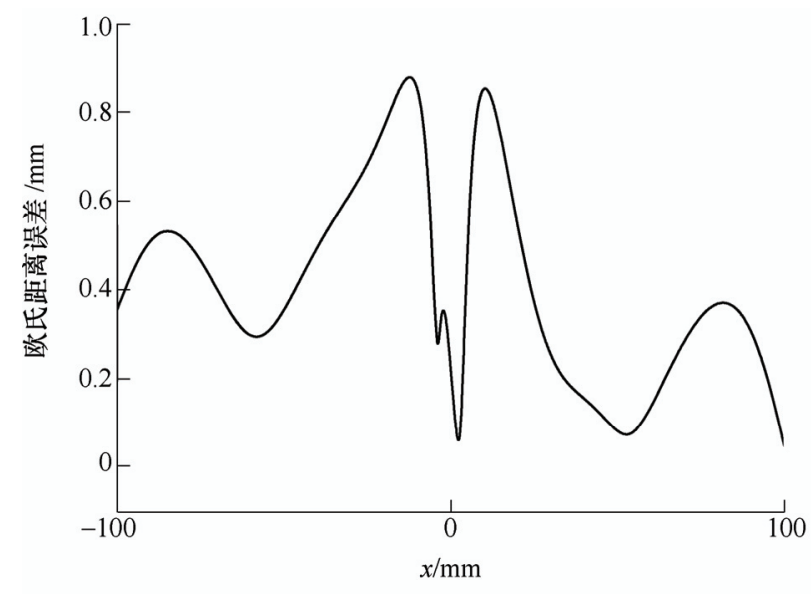

(b) 第一组综合结果误差曲线

图 8 应用算例第一组综合结果对比图及误差图

驱动角度下限为 $-40.3353^{\circ}$, 上限为 $40.5756^{\circ}$ 。最后, 激活模块中的传感器, 设置 $P$ 点到两个平面 $(y z$ 平面 和 $x z$ 平面)的测距值分别为输出曲线的横纵坐标, 对机构仿真模型的连杆轨迹曲线进行输出(图 9)。所 得轨迹曲线与目标轨迹曲线一致, 证明了机构模型 的正确性和轨迹综合方法的有效性。

根据仿真模型对实际滚轮进给机构进行搭建。 首先, 根据仿真模型参数对滚轮进给机构杆长及位
置进行调整, 调整后的滚轮进给机构如图 10a 所示, 机构简图如图 10b 所示。然后, 利用伺服控制器控 制电动机输出(ASD-A2-0721-F)。根据实际情况采用 定位控制, 转速设置为 $5 \mathrm{r} / \mathrm{min}$ 。最后, 分三次对目 标路径进行滚压包边试验, 滚压包边效果如图 $10 \mathrm{c}$ 所示, 其中, 第一次采用雉角为 $30^{\circ}$ 的滚头进行滚 压(图 10d), 第二次采用雉角为 $60^{\circ}$ 的滚头进行滚压 (图 10e), 第三次采用锥角为 $90^{\circ}$ 的滚头进行滚压 (图 10f)。通过试验结果可以看出, 所设计的进给机 构可以实现滚头的滚压路径, 进而完成滚压包边。 通过对实际机构的设计及试验验证了本文提出方法 的实用性, 同时可以为利用连杆机构代替工业机器 人实现滚压包边加工提供参考。

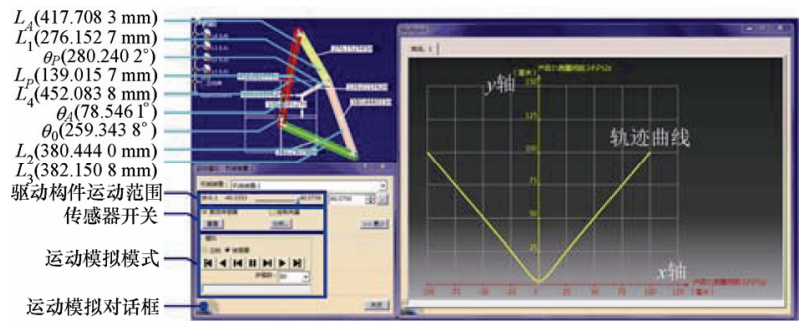

图 9 目标机构的 CATIA 仿真

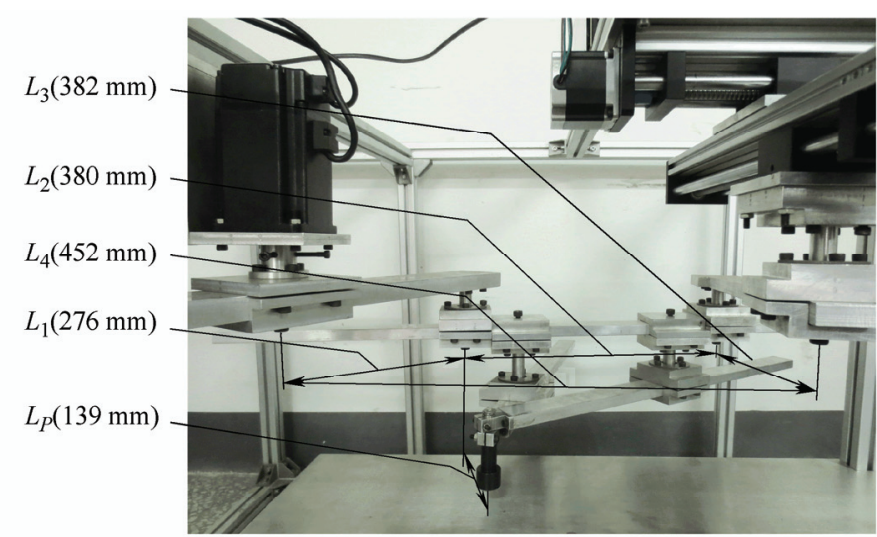

(a) 实际滚轮进给机构装配图

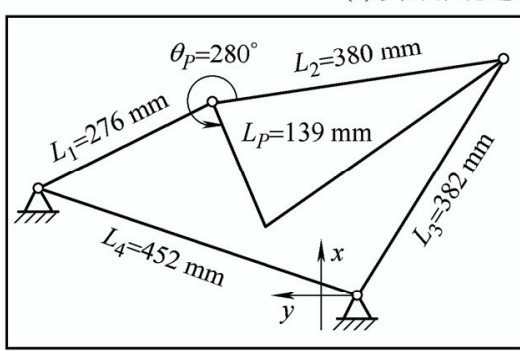

(b) 机构简图

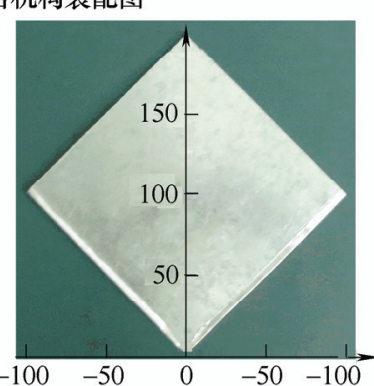

(c) 滚压包边效果图

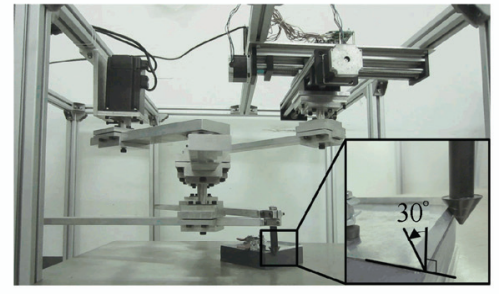

(d) $30^{\circ}$ 包边

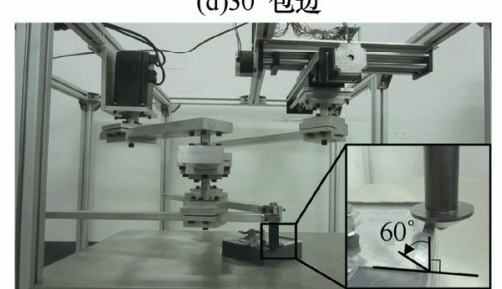

(e) $60^{\circ}$ 包边

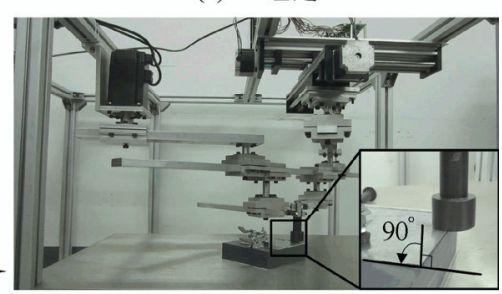

(f) $90^{\circ}$ 包边

图 10 滚压包边过程及滚压效果图

\section{4 结论}

（1）利用小波特征参数描述了平面四杆机构
连杆轨迹曲线特征，通过分析，发现机构尺寸型 与小波特征参数之间的内在联系，从而建立包含 3004281900 组平面四杆机构的机构尺寸型数据 库，实现相同机构尺寸型的聚类储存。克服了曲线 
图谱法检索效率低和图谱中包含尺寸型数量有限的 缺点; 弥补了傅里叶级数法无法对特定相对转动区 间进行轨迹综合的不足。

(2) 利用多维搜索树, 对 135 个相对转动区间 内的机构尺寸型进行分区。根据设计要求建立动态 自适应图谱库，实现了非预定相对转动区间的平面 四杆机构轨迹综合问题的求解。克服了精确点法存 在的给定精确位置个数限制的制约; 避免了近似综 合方法涉及的非线性方程组求解、优化初值选取和 迭代收敛性等问题。

（3）对滚压包边设备的滚轮进给机构进行设 计, 利用四杆机构取代工业机器人来带动滚轮实现 滚压包边。通过对实际机构的设计为滚压包边提供 新的思路。

\section{参 考 文 献}

[1] 张均富, 徐礼矩, 王杰. 可调球面六杆机构轨迹综合 [J]. 机械工程学报, 2007, 43(11): 50-55.

ZHANG Junfu, XU Liju, WANG Jie. Synthesis of adjustable spherical six-bar mechanisms for path generation[J]. Chinese Journal of Mechanical Engineering, 2007, 43(11): 50-55.

[2] 张新歌. 基于神经网络的铰链四杆机构复演轨迹设计 软件的开发[D]. 苏州: 苏州大学, 2008 .

ZHANG Xinge. Development of design software on generation of path curves with four-bar linkage mechanism based on neural networks[D]. Suzhou : Soochow University, 2008.

[3] WU J, GE Q J, GAO F, et al. Fourier descriptors with different shape signatures: A comparative study for shape based retrieval of kinematic constraints[J]. Chinese Journal of Mechanical Engineering, 2011, 24(5): 723-730.

[4] 孙晓斌, 肖人涁, 王雪. 基于曲线几何特征量化提取的 轨迹机构检索生成方法 [J]. 机械工程学报, 2000 , 36(11): 98-105.

SUN Xiaobin, XIAO Renbin, WANG Xue. New approach to rapid searching path generating mechanisms $[\mathrm{J}]$. Chinese Journal of Mechanical Engineering, 2000, 36(11): 98-105.

[5] SUN J W, LIU W R, CHU J K. Dimensional synthesis of open path generator of four-bar mechanisms using the Haar wavelet[J]. Journal of Mechanical Design, 2015, 137(8): 082303.

[6] 蓝兆辉, 邹慧君. 基于轨迹局部特性的机构并行优化综 合[J]. 机械工程学报, 1999，35(5): 16-19.

LAN Zhaohui, ZOU Huijun. Concurrent optimum synthesis of path generating mechanisms based on the local characteristics[J]. Chinese Journal of Mechanical
Engineering, 1999, 35(5): 16-19.

[7] YU H Y, TANG D W, WANG Z X. Study on a new computer path synthesis method of a four-bar linkage[J]. Mechanism and Machine Theory, 2007, 42(4): 383-392.

[8] UNRUH V, KRISHNASWAMI P. A Computer-aided design technique for semi-automated infinite point coupler curve synthesis of four-bar linkages[J]. ASME Journal of Mechanical Design, 1995，117(1): 143-149.

[9] 黄灿明, 郭海鹏, 陈永康, 等. 基于 B 样条插值曲线的 平面四杆机构轨迹复演优化设计 [J]. 机械工程学报, 1998, 34(3): 72-79.

HUANG Canming, GUO Haipeng, CHEN Yongkang, et al. Optimization scheme for four-bar mechanism path synthesis based on B-spline[J]. Chinese Journal of Mechanical Engineering, 1998，34(3): 72-79.

[10] 于红英, 赵彦微, 许栋铭. 平面铰链四杆机构的轨迹综 合方法[J]. 哈尔滨工业大学学报, 2015，47(1): 40-47. YU Hongying, ZHAO Yanwei, XU Dongming. A path synthesis method of planar hinge four-bar linkage[J]. Jouranl of Harbin Institute of Technology，2015， 47(1): 40-47.

[11] MCGARVA J R , MULlineUX G. Harmonic representation of closed curves[J]. Applied Mathematical Modelling, 1993, 17(4): 213-218.

[12] MCGARVA J R. Rapid search and selection of path generating linkages from a library[J]. Mechanism and Machine Theory, 1994, 29(2): 223-235.

[13］褚金奎, 孙建伟. 基于傅里叶级数理论的连杆机构轨迹 综合方法[J]. 机械工程学报, 2010, 46(13): 31-41. CHU Jinkui, SUN Jianwei. Unified approach to synthesis of coupler curves of linkage by Fourier series[J]. Journal of Mechanical Engineering, 2010，46(13): 31-41.

[14] SUN J W, CHU J K. A unified model of harmonic characteristic parameter method for dimensional synthesis of linkage mechanism[J]. Applied Mathematical Modelling, 2012, 36(12): 6001-6010.

[15] MULLINEUX G. Atlas of spherical four-bar mechanisms $[\mathrm{J}]$. Mechanism and Machine Theory, 2011, 46(11): 1811-1823.

[16] WU J, GE Q J, GAO F, et al. On the extension of a Fourier descriptor based method for planar four-bar linkage synthesis for generation of open and closed paths[J]. Journal of Mechanisms and Robotics, 2011, 3(3): 031002.

[17] YUE C, SU H J, GE Q J. A hybrid computer-aided linkage design system for tracing open and closed planar curves[J]. Computer-Aided Design, 2012，44(11): 1141-1150.

[18] 吴金金, 褚金奎, 曹惟庆. 平面四杆机构函数输出的小波 变换分析 $[\mathrm{J}]$. 机械科学与技术，1998，17(2): 180-182. 
WU Xin, CHU Jinkui, CAO Weiqing. Analysis of output function of 4-bar linkages using wavelet transform. Mechanical Science and Technology, 1998, 17(2): 180-182.

[19] 王成志, 纪跃波, 孙道恒. 小波分析在平面四杆机构轨 迹综合中的应用研究 [J]. 机械工程学报, 2004, 40(8): 34-39.

WANG Chengzhi, JI Yuebo, SUN Daoheng. Research on applying wavelet analysis to path synthesis for coupler curves of planar four-bar linkages[J]. Chinese Journal of Mechanical Engineering, 2004, 40(8): 34-39.

[20] GALÁN-MARÍN G, ALONSO F J, CASTILlO J M D. Shape optimization for path synthesis of crank-rocker mechanisms using a wavelet-based neural network[J]. Mechanism and Machine Theory, 2009, 44(6): 1132-1143.

[21] SUN J W, LIU W R, CHU J K. Synthesis of spherical four-bar linkage for open path generation using wavelet feature parameters $[\mathrm{J}]$. Mechanism and Machine Theory, 2018, 128: 33-46.

[22] LIU W R, SUN J W, ZHANG B C, et al. Wavelet feature parameters representations of open planar curves[J]. Applied Mathematical Modelling, 2018, 57: 614-624.

[23] LIU S Z, DAI J S , LI A M, et al. Analysis of frequency characteristics and sensitivity of compliant mechanisms[J]. Chinese Journal of Mechanical Engineering, 2016, 29(4): 1-14.

[24] 陈放, 李欣玲, 余霞, 等. 平面轨迹机构时变可靠性分 析的联合概率方法 [J]. 机械工程学报， 2017，53(15): 119-124

CHEN Fang, LI Xinling, SHE Xia, et al. Joint probability method to time-dependent reliability for planar path mechanisms[J]. Journal of Mechanical Engineering, 2017, 53(15): 119-124.

[25] 郑国峰, 上官文斌, 韩鹏飞, 等. 基于小波变换的汽车 零部件加速耐久性载荷谱编辑方法研究 $[\mathrm{J}]$. 机械工程 学报, 2017, 53(8): 124-131.

ZHENG Guofeng, SHANGGUAN Wenbin, HAN Pengfei, et al. Study of load spectrum edition method based on the wavelet transform to the accelerated durability test of the vehicle component[J]. Journal of Mechanical Engineering, 2017， 53(8): 124-131.

[26] 孙殿柱, 郭洪帅, 李延瑞, 等. 基于局部泊松曲面重建 的点云刚性配准方法 [J]. 机械工程学报, 2018, 54(15): 141-149.

SUN Dianzhu, GUO Hongshuai, LI Yanrui, et al. Method of rigid registration based on Poisson reconstruction of local sample points[J]. Journal of Mechanical Engineering, 2018, 54(15): 141-149.

[27] CHEN D K, DING J J, GAO M Z, et al. Form gene clustering method about pan-ethnic-group products based on emotional semantic[J]. Chinese Journal of Mechanical Engineering, 2016, 29(6): 1134-1144.

作者简介: 刘文瑞, 男, 1989 年出生, 博士研究生。主要研究方向为机 器人机构学、连杆机构分析与综合

E-mail: wenruilv@126.com

孙建伟(通信作者), 男, 1979 年出生, 博士, 教授, 博士研究生导师。 主要研究方向为机器人机构学、仿生机构学、连杆机构分析与综合。

E-mail: avensun@tom.com

褚金奎，男，1965 年出生，博士，教授，博士研究生导师。主要研究方 向为微机械电子系统、仿生微纳传感器与执行器、机器人机构学及 CAD、 智能机器人及微机器人。

E-mail: chujk@dlut.edu.cn 